\title{
Using a Dual-BEM formulation to model the sound pressure wavefield provided by absorbing thin screens attached to the walls of a duct
}

\author{
J. António, L. Godinho, P. Amado Mendes \& A. Tadeu \\ Department of Civil Engineering, University of Coimbra, Portugal
}

\begin{abstract}
The modelling of the 2D sound pressure wavefield in the presence of thin absorbing screens attached to the walls of a duct, assumed to be infinitely long, is described in this paper. The simulation uses a frequency-domain Dual-BEM (BEM/TBEM) formulation, which overcomes the thin-body difficulty that arises with the classical BEM formulation. The sound absorption is simulated by imposing impedance boundary conditions, which are applied in conjunction with a Dual-BEM approach. The fundamental solutions used in the formulation allow the solution to be obtained without discretizing the duct's walls. Thus, only the boundary of each absorbing screen attached to the duct's walls is modelled, which makes the proposed formulation efficient even at high excitation frequencies. The hypersingular integrals that result from the implementation of the TBEM are computed analytically. The formulation is used to compare results obtained with absorbing screens with those obtained with rigid screens and with those obtained in a duct without screens. Results in the time domain are obtained by applying an inverse Fourier transform to the frequency results.

Keywords: dual-BEM, thin body, sound absorption.
\end{abstract}

\section{Introduction}

The demand for quieter environments has led to the imposition of lower noise levels inside buildings. Those limits are usually imposed by legislation and building codes. Sound pressure levels inside buildings are often reduced by installing sound attenuation systems in ventilation ducts. 
The usual strategies for noise attenuation in ducts consist of fitting Helmholtz resonators, and lined expansion chambers. However, these devices tend to occupy too much space which is a drawback if there are space limitations. Most studies in this field have analysed and/or modelled the effect of placing absorptive linings on the walls of the duct [1] or applying some sort of expansion chamber to reduce the noise in the duct [2].

A two-dimensional analytical solution was developed by Selamet et al. [3] to determine the acoustic performance of a perforated single-pass, concentric cylindrical silencer filled with fibrous material. Lawrie and Guled [4] and Huang [5] developed modal approaches to investigate the performance of modified reactive silencers in which a membrane is attached to the internal walls of an expansion chamber.

The coupling between modal methods and the finite element method has been used to model sound propagation in ductwork that contains one or more nonuniform obstacles [2]. A general finite element formulation for the analysis of the sound field in a flow duct with a surrounding space filled with porous or fibrous material was proposed by Peat and Rathi [6]. Boundary element methods (BEM) and analytical methods have been employed by Selamet et al. [7] to predict the acoustic attenuation of a hybrid silencer that consists of two singlepass perforated filling chambers and a Helmholtz resonator.

As some of these systems cannot be used where there are space constraints several authors have considered installing noise screens within the duct. Rim and Kim [8], for instance, analysed experimentally and analytically the attenuation produced by acoustic screens inside a rectangular ventilation duct. Our work follows a similar principle and sets out to simulate the sound wave propagation inside a duct where the sound attenuation is provided by thin elements working as screens to counter sound propagation.

The boundary element method has been widely used to model acoustic problems $[9,10]$. The major advantage of this method is that it is particularly effective for modeling unconfined media since it automatically satisfies the farfield conditions, and only requires the discretization of the interfaces of the objects to be modelled. But it has some limitations when used to model objects of very small thickness because the implementation of the integral equations on both sides of the object leads to a singular system of equations.

This problem can be solved if the Dual Boundary Element Method (DualBEM) is applied [11]. It uses the classic formulation of the boundary integral equation on one side of the object and its first spatial derivative along the direction orthogonal to the boundary on the opposite side, to yield a system of equations that is not singular.

When the object has null thickness a very efficient approach is the traction boundary element method formulation (TBEM). In this case the object to be discretized is represented by only one line of boundary elements and the resulting system of equations is smaller than that provided by the Dual-BEM formulation $[12,13]$. These methods have been employed to solve problems of sound propagation in the presence of rigid noise barriers over a rigid or an impedance plane $[11,13]$. 
In this work a Dual-BEM formulation is applied in the frequency domain to simulate the sound propagation in a duct in the presence of two-dimensional thin screens with an absorbing surface. This formulation includes fundamental solutions for a fluid layer with rigid boundaries [12], using the technique of image sources. Only the screens' surfaces need to be discretized, not the duct boundaries. A given impedance (Robin boundary condition) [14] is taken as a boundary condition in the Dual-BEM formulation to ascribe sound absorption to the screens' surface. The sound absorption coefficients of the boundaries are thus taken into account by introducing impedance coefficients that are defined by the ratio between the pressure and velocity of the sound waves that propagate from the source to each nodal point, considering a coefficient of reflection for the incident waves. This formulation lets the absorption coefficients be frequency dependent and also allows the screens to be modelled with any irregular geometry. The problem formulation and the Dual-BEM approach are presented next, followed by a numerical application to illustrate the applicability of the method.

\section{Problem definition}

The problem consists of an infinitely long fluid layer, with rigid boundaries, of density $\rho$, in which the waves propagate with a speed $c$, where a twodimensional cylindrical inclusion limited by a surface $S$ is embedded (Figure 1).

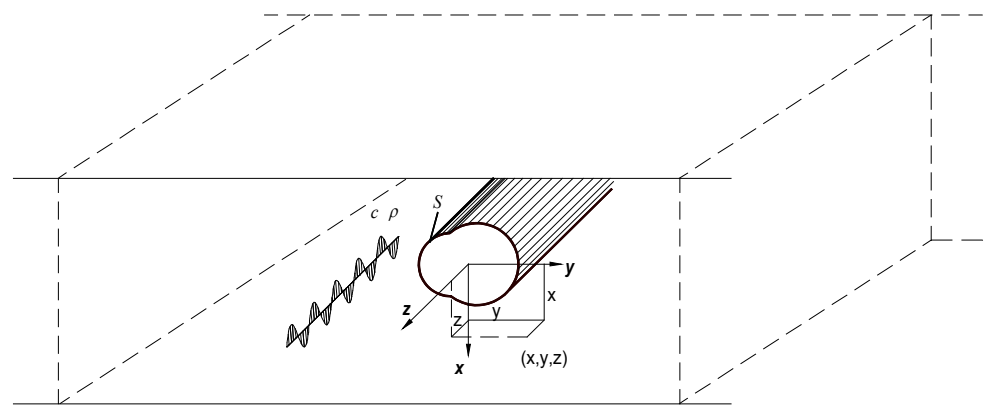

Figure 1: Problem definition.

When this system is subjected to a pressure line source placed at $\left(x_{s}, y_{s}\right)$ emitting at a frequency $\omega$, whose amplitude varies sinusoidally in the third dimension $(z)$, the incident sound pressure generated in the frequency domain can be expressed as:

$$
p_{\text {inc }}\left(x, y, x_{s}, y_{s}, k_{z}, \omega\right)=H_{0}\left(k_{c} r_{0}\right) \mathrm{e}^{-\mathrm{i} k_{z} z},
$$


where $H_{n}(\ldots)$ are second kind Hankel functions of the order $n, \mathrm{i}=\sqrt{-1}$ and $k_{c}=\sqrt{\frac{\omega^{2}}{c^{2}}-k_{z}^{2}}$, with $\operatorname{Im}\left(k_{c}\right)<0, r_{0}=\sqrt{\left(x-x_{s}\right)^{2}+\left(y-y_{s}\right)^{2}}$, and $k_{z}$ is the axial wavenumber.

The pressure $(p)$ at any point in the domain can be calculated with the Helmholtz equation:

$$
\left(\frac{\partial^{2}}{\partial x^{2}}+\frac{\partial^{2}}{\partial y^{2}}\right) p\left(x, y, k_{z}, \omega\right)+\left(k_{c}\right)^{2} p\left(x, y, k_{z}, \omega\right)=0 .
$$

\section{Boundary element method formulation}

This section describes the boundary integral formulations needed to calculate the pressure wavefield scattered by the inclusion.

The objective is to model screens of small thickness within an infinitely long fluid layer simulating a duct. The classical formulation of the boundary element method degenerates when used to calculate the wavefield scattered by a thin inclusion. The problem can be solved by means of a Dual-BEM approach that consists of applying the BEM and TBEM formulations at opposite collocation points of the discretized boundary. The application of the BEM formulation to the surface is equivalent to the use of monopole loads while the application of the TBEM formulation is equivalent to the use of dipole loads.

\subsection{BEM formulation}

The boundary integral equation used in the classical formulation of the BEM can be derived from the Helmholtz equation in the frequency domain by applying the reciprocity theorem:

$$
\begin{aligned}
& b p\left(x_{0}, y_{0}, k_{z}, \omega\right)=\int_{S} q\left(x, y, \mathbf{n}_{n 1}, k_{z}, \omega\right) G\left(x, y, x_{0}, y_{0}, k_{z}, \omega\right) \mathrm{d} s- \\
& \int_{S} H\left(x, y, \mathbf{n}_{n 1}, x_{0}, y_{0}, k_{z}, \omega\right) p\left(x, y, k_{z}, \omega\right) \mathrm{d} s+p_{\text {inc }}\left(x_{0}, y_{0}, x_{s}, y_{s}, k_{z}, \omega\right),
\end{aligned}
$$

where $G$ and $H$ are fundamental solutions for pressure $(p)$ and pressure gradients $(q)$ at $(x, y)$ on the boundary $S$ due to a virtual point pressure source at a collocation point $\left(x_{0}, y_{0}\right) . \mathbf{n}_{n_{1}}$ is the unit outward normal along the boundary $S$, at $(x, y)$, defined by the vector $\mathbf{n}_{n 1}=\left(\cos \theta_{n 1}, \sin \theta_{n 1}\right)$. The factor $b$ takes the value $1 / 2$ if $\left(x_{0}, y_{0}\right) \in S$ and $S$ is smooth. 
The fundamental solutions for pressure and pressure gradients in Cartesian coordinates for an infinite medium are:

$$
\begin{gathered}
G\left(x, y, x_{0}, y_{0}, k_{z}, \omega\right)=-\frac{\mathrm{i}}{4} H_{0}\left(k_{c} r_{01}\right) \\
H\left(x, y, \mathbf{n}_{n 1}, x_{0}, y_{0}, k_{z}, \omega\right)=\frac{\mathrm{i}}{4} k_{c} H_{1}\left(k_{c} r_{01}\right) \frac{\partial r_{01}}{\partial \mathbf{n}_{n 1}},
\end{gathered}
$$

with $r_{01}=\sqrt{\left(x-x_{0}\right)^{2}+\left(y-y_{0}\right)^{2}}$.

\subsection{TBEM formulation}

The boundary integral equation formulated in tractions can be derived by applying the gradient operator to the boundary integral equation (3), which represents the application of dipole pressure loads. In this case, when the surface of the boundary is loaded by dipole loads, the boundary integral equation in tractions can be written as follows:

$$
\begin{aligned}
& a p\left(x_{0}, y_{0}, k_{z}, \omega\right)+b q\left(x_{0}, y_{0}, \mathbf{n}_{n 1}, k_{z}, \omega\right)= \\
& \int_{S} q\left(x, y, \mathbf{n}_{n 1}, k_{z}, \omega\right) \bar{G}\left(x, y, \mathbf{n}_{n 2}, x_{0}, y_{0}, k_{z}, \omega\right) d s- \\
& \int_{S} \bar{H}\left(x, y, \mathbf{n}_{n 1}, \mathbf{n}_{n 2}, x_{0}, y_{0}, k_{z}, \omega\right) p\left(x, y, k_{z}, \omega\right) d s+\bar{p}_{i n c}\left(x_{0}, y_{0}, \mathbf{n}_{n 2}, x_{s}, y_{s}, k_{z}, \omega\right)
\end{aligned}
$$

The fundamental solutions $\bar{G}$ and $\bar{H}$ are calculated by deriving the initial fundamental solutions $G$ and $H$. In this equation, $\mathbf{n}_{n 2}$ is the unit outward normal to the boundary $S$ at the collocation points $\left(x_{0}, y_{0}\right)$, defined by the vector $\mathbf{n}_{n 2}=\left(\cos \theta_{n 2}, \sin \theta_{n 2}\right)$. In equation (5) $a$ takes a null value for piecewise straight boundary elements and the fundamental solutions for an unbounded medium are defined as follows:

$$
\begin{aligned}
& \bar{G}\left(x, y, \mathbf{n}_{n 2}, x_{0}, y_{0}, k_{z}, \omega\right)=\frac{\mathrm{i}}{4} k_{c} H_{1}\left(k_{c} r_{01}\right) \frac{\partial r_{01}}{\partial \mathbf{n}_{n 2}} \\
& \bar{H}\left(x, y, \mathbf{n}_{n 1}, \mathbf{n}_{n 2}, x_{0}, y_{0}, k_{z}, \omega\right)= \\
& \frac{\mathrm{i}}{4} k_{c}\left\{-k_{c} H_{2}\left(k_{c} r_{01}\right)\left[\left(\frac{\partial r_{01}}{\partial x}\right)^{2} \frac{\partial x}{\partial \mathbf{n}_{n 1}}+\frac{\partial r_{01}}{\partial x} \frac{\partial r_{01}}{\partial y} \frac{\partial y}{\partial \mathbf{n}_{n 1}}\right]+\frac{H_{1}\left(k_{c} r_{01}\right)}{r_{01}}\left[\frac{\partial x}{\partial \mathbf{n}_{n 1}}\right]\right\} \frac{\partial x}{\partial \mathbf{n}_{n 2}}+ \\
& \frac{\mathrm{i}}{4} k_{c}\left\{-k_{c} H_{2}\left(k_{c} r_{01}\right)\left[\frac{\partial r_{01}}{\partial x} \frac{\partial r_{01}}{\partial y} \frac{\partial x}{\partial \mathbf{n}_{n 1}}+\left(\frac{\partial r_{01}}{\partial y}\right)^{2} \frac{\partial y}{\partial \mathbf{n}_{n 1}}\right]+\frac{H_{1}\left(k_{c} r_{01}\right)}{r_{01}}\left[\frac{\partial y}{\partial \mathbf{n}_{n 1}}\right]\right\} \frac{\partial y}{\partial \mathbf{n}_{n 2}}
\end{aligned}
$$

The incident field in equation (5) is given by:

$$
\bar{p}_{i n c}\left(x_{0}, y_{0}, \mathbf{n}_{n 2}, x_{s}, y_{s}, k_{z}, \omega\right)=-k_{c} H_{1}\left(k_{c} r_{0}\right)\left(\frac{\partial r_{0}}{\partial x} \frac{\partial x}{\partial \mathbf{n}_{n 2}}+\frac{\partial r_{0}}{\partial y} \frac{\partial y}{\partial \mathbf{n}_{n 2}}\right) .
$$


In the present formulations only the surface of the screens is discretized by boundary elements. Thus, in each formulation the fundamental solutions take into account terms that are obtained by the technique of image sources [12], with respect to the waves reflected by the horizontal boundaries of the duct.

\subsection{Sound absorption simulation}

The imposition of absorption on the screens' surface is simulated by relating the velocity and pressure at each nodal point as follows:

$$
q\left(x, y, \mathbf{n}_{n 1}, k_{z}, \omega\right)=-\mathrm{i} \omega \rho \frac{1}{\mathrm{Z}(\omega)} p\left(x, y, k_{z}, \omega\right)
$$

to be used in equations (3) and (5).

The impedance factor is expressed by the ratio between the velocity and pressure and the sound absorption coefficient $\alpha$ through the expression,

$$
Z(\omega)=\frac{p_{\text {inc }}\left(x_{0}, y_{0}, x_{s}, y_{s}, k_{z}, \omega\right)}{v_{\text {inc }}\left(x_{0}, y_{0}, x_{s}, y_{s}, \mathbf{n}_{n 1}, k_{z}, \omega\right)}\left(\frac{1+\sqrt{1-\alpha}}{1-\sqrt{1-\alpha}}\right),
$$

where $p_{\text {inc }}\left(x_{0}, y_{0}, x_{s}, y_{s}, k_{z}, \omega\right)=\mathrm{H}_{0}\left(k_{c} r_{00}\right) e^{-\mathrm{i} k_{z} z}$

$$
v_{i n c}\left(x_{0}, y_{0}, x_{s}, y_{s}, \mathbf{n}_{n 1}, k_{z}, \omega\right)=-k_{c} H_{1}\left(k_{c} r_{00}\right) e^{-\mathrm{i} k_{z} z} \frac{\partial r_{00}}{\partial \mathbf{n}_{n 1}}
$$

are the incident pressure and velocity respectively, with $r_{00}=\sqrt{\left(x_{0}-x_{s}\right)^{2}+\left(y_{0}-y_{s}\right)^{2}}$.

The solution of the equations (3) and (5) requires the discretization of the surface $S$ with $N$ straight boundary elements with a nodal point in the centre of each element. The required integrations are performed using Gaussian quadrature when the loaded element is to be integrated. When the element to be integrated is the loaded element the integrals become hypersingular and must be solved analytically $[15,16]$.

\section{Time domain pressure wavefield}

The model allows the calculation, in the frequency domain, of the sound pressure at any point of the medium. The time variation of the pressure wavefield can be obtained by applying an inverse Fourier transform to the frequency domain findings. The temporal pressure variation is modelled by having the source modelled as a Ricker pulse of a given characteristic frequency.

The Ricker pulse is defined in the frequency domain by

$$
U(\omega)=A\left[2 \sqrt{\pi} t_{o} \mathrm{e}^{-\mathrm{i} \omega t_{s}}\right] \Omega^{2} \mathrm{e}^{-\Omega^{2}},
$$


where $A$ is the amplitude, $\Omega=\omega t_{o} / 2, t_{s}$ is the instant at which the function reaches its maximum and $\pi t_{o}$ is the characteristic period of the function.

The simulations allow the observation of the event for a period that depends on the frequency increment $\Delta \omega$, and is equal to $T=2 \pi / \Delta \omega$. For a given receiver, if the event lasts longer than the time modelled aliasing phenomena arise. The pulses arriving at the receiver after $T$ will appear at the initial instants. This is avoided by introducing a response attenuation through complex frequencies with a small imaginary part of the form $\omega_{c}=\omega-\mathrm{i} \eta$ (with $\eta=0.7 \Delta \omega)$ [17]. This artificial effect is removed in the time domain by rescaling the response using an exponential window $\mathrm{e}^{\eta t} \quad[18]$.

\section{Application of the model}

The applicability of the numerical formulation just described is illustrated by modeling acoustic screens inside a duct of infinite extent.

The $2 \mathrm{D}$ pressure wavefield is calculated in an air layer $\left(\rho=1.22 \mathrm{~kg} \mathrm{~m} \mathrm{~m}^{3}\right)$ confined by rigid boundaries (duct) in which screens of reduced thickness are located. The duct is $0.30 \mathrm{~m}$ thick and contains six screens of $1 \mathrm{~mm}$ thickness at an inclination of 20 degrees in relation to the vertical direction (see Figure 2).

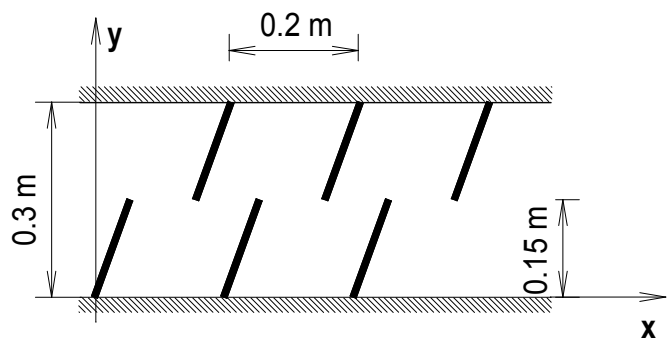

Figure 2: Geometry of the problem.

The boundaries of the screens are discretized by boundary elements whose number varies depending on the frequency of the source. A ratio equal to ten is adopted between the wavelength of the incident waves and the length of the boundary elements. The minimum number of boundary elements used in each screen was 48.

A pressure line load $\left(\mathrm{k}_{\mathrm{z}}=0.0 \mathrm{rad} / \mathrm{m}\right)$ located at $\mathrm{x}=-0.2 \mathrm{~m}$ and $\mathrm{y}=0.15 \mathrm{~m}$ disturbs the air medium, where the pressure fluctuation is registered at a grid of 9000 receivers.

The computations are performed in the frequency range of $[200 \mathrm{~Hz}$, $25600 \mathrm{~Hz}$ ], with a frequency increment of $200 \mathrm{~Hz}$, which defines the total time duration of $5 \mathrm{~ms}$. The pressure in the time domain is obtained by applying an inverse Fourier transform to the frequency results. The source is modelled as a Ricker pulse with a characteristic frequency of $8000 \mathrm{~Hz}$. 
To illustrate the effect of sound absorption in the pressure field, a constant absorption coefficient $\alpha=0.7$ at all frequencies was ascribed to the screen surfaces. Three simulations are presented to verify the effect of the screens and of the sound absorption coefficient on the pressure wavefield: a) the first considers only the duct without any obstacle inside; b) the second assumes the presence of rigid screens inside the duct; c) in the third the duct contains absorbing screens with a sound absorption coefficient of 0.7 .

A sequence of snapshots that display the pressure wave field over the grid of receivers at different instants is presented to illustrate the differences between these three scenarios. The pressure amplitude is displayed by a colour scale which ranges from blue to red as the amplitude increases.

At $t=0.77 \mathrm{~ms}$ (see Figure 3), the incident wavefront has been reflected on the walls of the duct. Where the screens are in place a first reflection on the surface of the first screen and a diffraction starting at the top of the screen are visible. It can also be seen that the reflected wave amplitude at the screen in Figure 3c) is lower than in Figure 3b), due to the sound absorption effect introduced in Figure 3c).

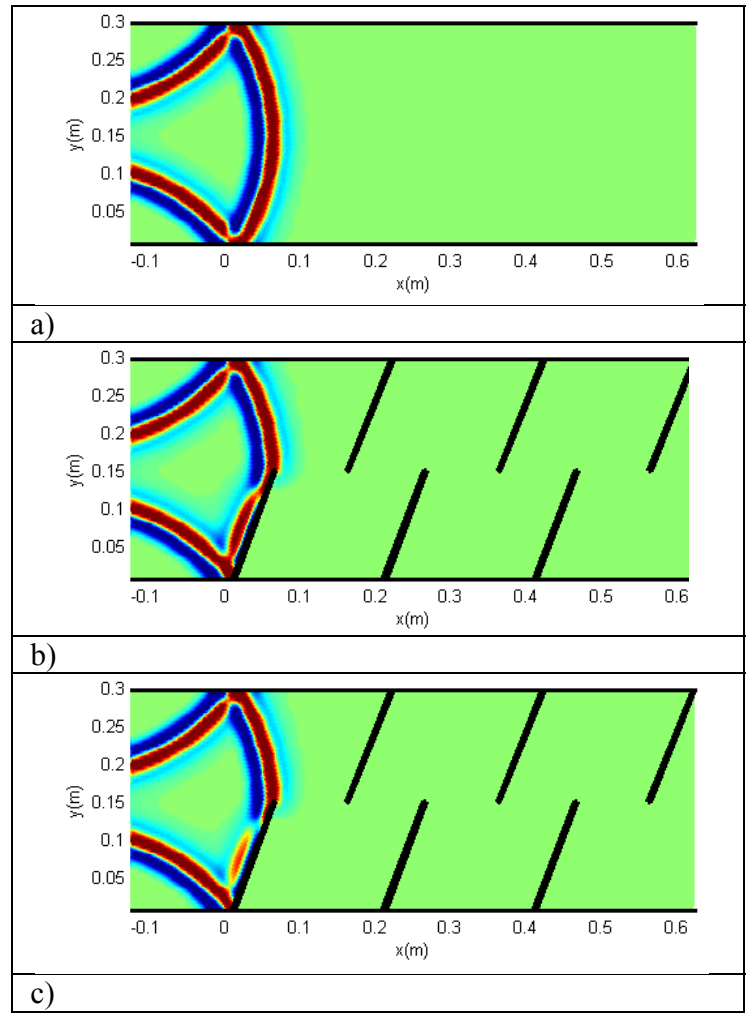

Figure 3: Time pressure responses over a grid of receivers for a characteristic frequency of $8000 \mathrm{~Hz}$, at $t=0.77 \mathrm{~ms}$ : a) duct; b) duct with rigid screens; c) duct with absorbing screens. 
Figure 4 shows that the pressure wavefield at the bottom of the duct between the first two lower screens (Figures $4 b$ ) and $4 c$ )) is more attenuated than the example without barriers (Figure 4a)). This is because the wave was reflected back by the first barrier.

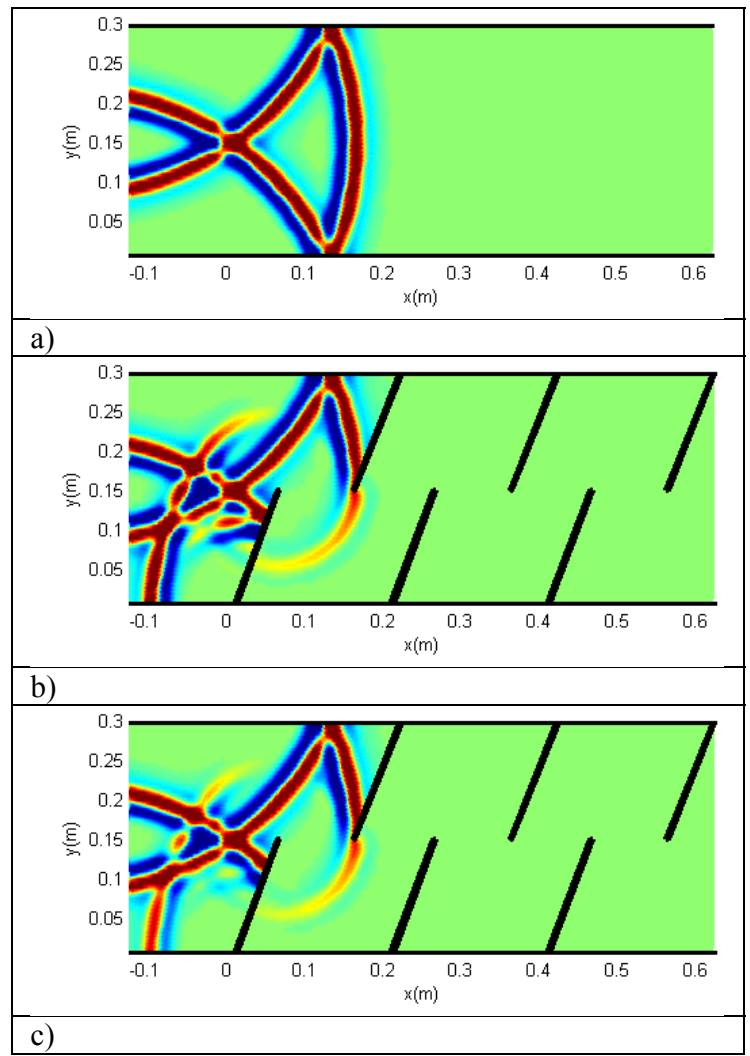

Figure 4: Time pressure responses over a grid of receivers for a characteristic frequency of $8000 \mathrm{~Hz}$, at $t=1.07 \mathrm{~ms}$ : a) duct; b) duct with rigid screens; c) duct with absorbing screens.

At $t=1.40 \mathrm{~ms}$ a wave reflection on the first upper screen and the wavefront reflection and diffraction on the second lower screen are visible (Figures 5b) and 5c)). The amplitude of these waves (near the second lower screen) is low due to the energy that was reflected back by the previous screens. A slight decrease in the amplitude of Figure 5c) in relation to Figure 5b) is also detectable; it is caused by the presence of absorption in the screens of Figure $5 \mathrm{c}$ ). 


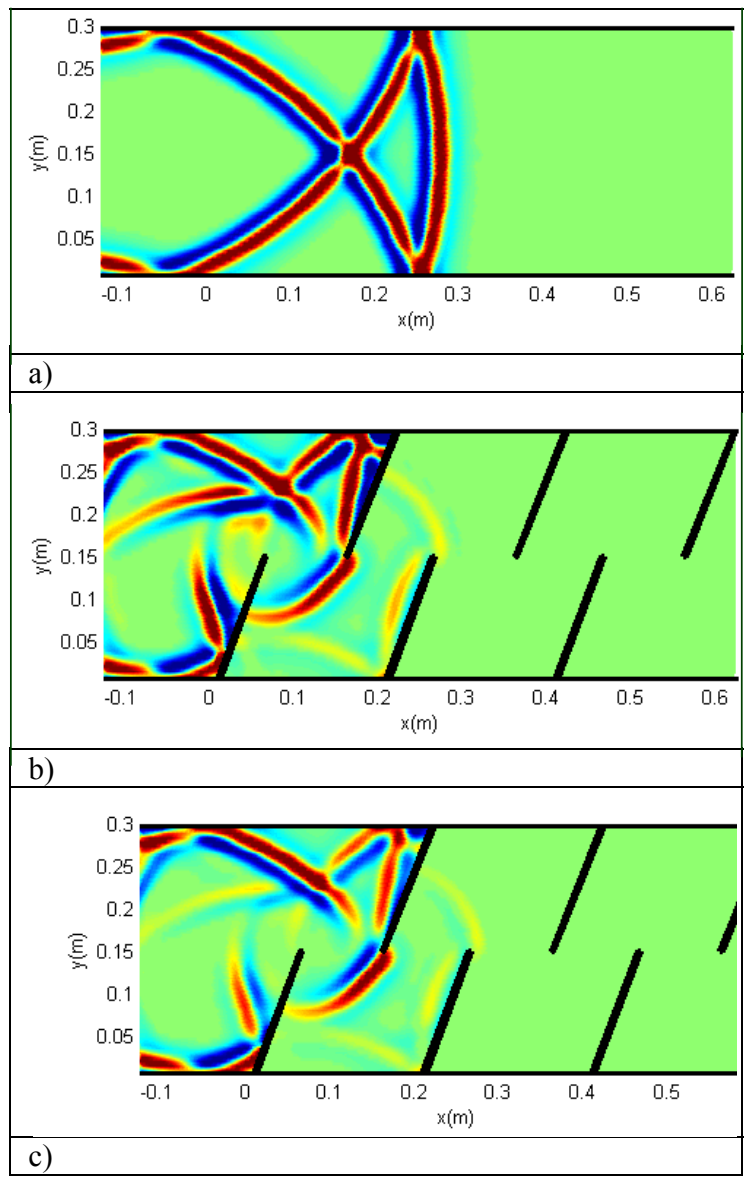

Figure 5: Time pressure responses over a grid of receivers for a characteristic frequency of $8000 \mathrm{~Hz}$, at $t=1.40 \mathrm{~ms}$ : a) duct; b) duct with rigid screens; c) duct with absorbing screens.

As time elapses the wavefront's progress is obstructed by the screens, as can be seen by comparing Figure $6 a$ ) with Figures $6 b$ ) and $6 c$ ); in Figures $6 b$ ) and $6 c$ ) the waves are reflected back and also suffer successive reflections between screens. These multiple reflections have the disadvantage of enhancing the sound pressure field between screens (see Figure 6b)). However, this amplitude can be attenuated when the screens have absorption properties (see Figure 6c)). This effect is noticeable, for example, when observing the pressure field between the first and second lower screens in Figures 6b) and 6c) (a similar effect is seen between the first two upper screens). 


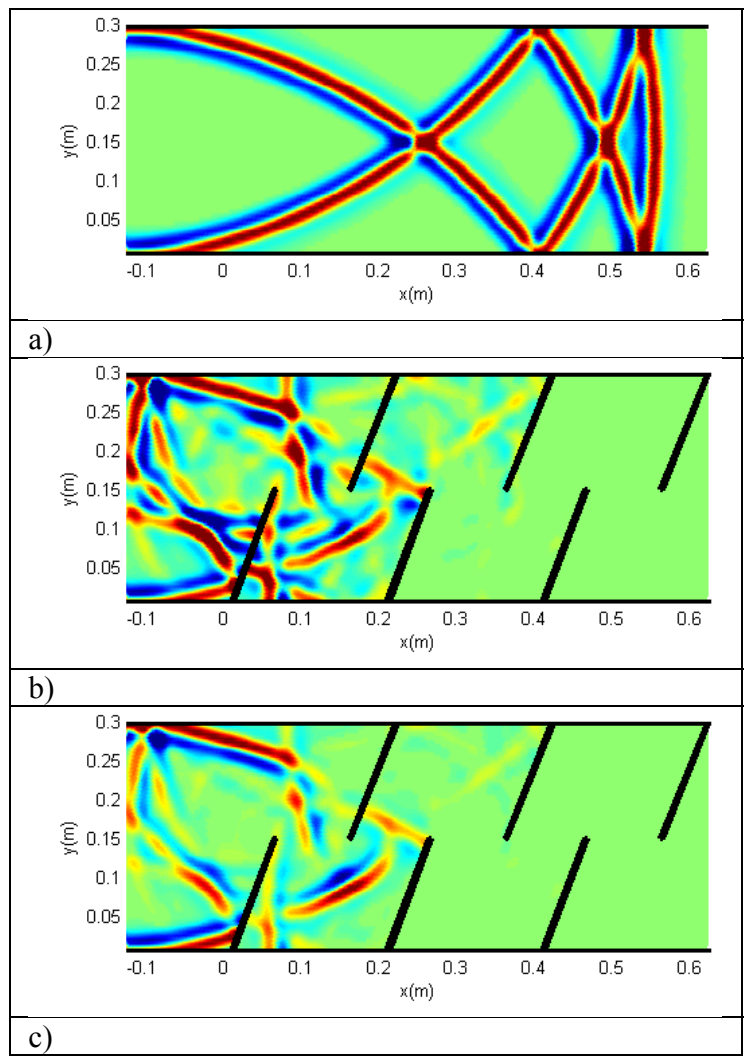

Figure 6: Time pressure responses over a grid of receivers for a characteristic frequency of $8000 \mathrm{~Hz}$, at $t=2.22 \mathrm{~ms}$ : a) duct; b) duct with rigid screens; c) duct with absorbing screens.

\section{Conclusions}

Wave propagation in an infinitely long air duct containing absorbing thin screens was modelled using a Dual formulation of the BEM. Sound absorption was included by considering a specific impedance at the boundaries of the barriers. Analysis of the results shows that the present model adequately simulates the physical phenomena involved. The insertion of rigid screens within the duct reduces the progress of the wavefront, and consequently the pressure amplitude along the duct, because energy is reflected back or trapped between the screens. However, since these reflections will increase the energy before and between screens the solution is to use sound absorption on the surface of the screens in order to reduce the sound field in those areas. 


\section{Acknowledgment}

The authors acknowledge the Instituto de Investigação e Desenvolvimento Tecnológico em Ciências da Construção - ITeCons for the support provided during the development of this work.

\section{References}

[1] Panigrahi, S.N. and Munjal, M.L., Comparison of various methods for analyzing lined circular ducts. Journal of Sound and Vibration, 285, pp. 905-923, 2005.

[2] Kirby, R., Modeling sound propagation in acoustic waveguides using a hybrid numerical method. Journal of the Acoustical Society of America, 124, pp. 1930-1940, 2008.

[3] Selamet, A., Xu, M.B. and Lee, I.-J., Analytical approach for sound attenuation in perforated dissipative silencers. Journal of the Acoustical Society of America, 115, pp. 2091-2099, 2004.

[4] Lawrie, J.B. and Guled, I.M.M., On tuning a reactive silencer by varying the position of an internal membrane. Journal of the Acoustical Society of America, 120, pp. 780-790, 2006.

[5] Huang, L., Modal analysis of a drumlike silencer. Journal of the Acoustical Society of America, 112, pp. 2014-2025, 2002.

[6] Peat, K.S. and Rathi, K.L., A finite element analysis of the convected acoustic wave motion in dissipative silencers. Journal of Sound and Vibration, 184, pp. 529-545, 1995.

[7] Selamet, A., Lee, I.J. and Huff, N.T., Acoustic attenuation of hybrid silencers. Journal of Sound and Vibration, 262, pp. 509-527, 2003.

[8] Rim, M. and Kim, Y.-H., Narrowband noise attenuation characteristics of in-duct acoustic screens. Journal of Sound and Vibration, 234, pp. 737-759, 2000.

[9] T. Wu [Ed.], Boundary Element Acoustics, WIT Press, Southampton, UK, 2000.

[10] Dawson, T.W. and Fawcett, J.A., A boundary integral equation method for acoustic scattering in a waveguide with nonplanar surfaces. Journal of the Acoustical Society of America, 87, pp. 1110-1125, 1990.

[11] de Lacerda, L. A., Wrobel, L. C. and Mansur, W. J., A dual boundary element formulation for sound propagation around barriers over an impedance plane. Journal of Sound and Vibration, 202, pp. 235-247, 1997.

[12] Antonio, J., Tadeu, A. and Amado Mendes, P., A 2.5D traction boundary element method formulation applied to the study of wave propagation in a fluid layer hosting a thin rigid body. Journal of Computational Acoustics, 16, pp. 177-198, 2008.

[13] Tadeu, A., António, J., Amado Mendes, P. and Godinho, L., Sound pressure level attenuation provided by thin rigid screens coupled to tall buildings. Journal of Sound and Vibration, 304, 479-496, 2007. 
[14] António, J., Tadeu, A. and Godinho, L., A Three-dimensional Acoustics Model using the Method of Fundamental Solutions. Engineering Analysis with Boundary Elements, 32, pp. 525-531, 2008.

[15] Tadeu, A., Santos, P.F.A. and Kausel, E., Closed-form integration of singular terms for constant, linear and quadratic boundary elements. Part I. SH wave propagation. Engineering Analysis with Boundary Elements, 23, pp. 671-681, 1999.

[16] Tadeu, A., António, J. and Castro, I., Coupling the BEM/TBEM and the MFS for the numerical simulation of acoustic wave propagation. Engineering Analysis with Boundary Elements, 34, pp. 405-416, 2010.

[17] Phinney, R., Theoretical calculation of the spectrum of first arrivals in layered elastic mediums. Journal of Geophysical Research, 70, pp. 5107$5123,1965$.

[18] Kausel, E. and Roesset, J.M., Frequency domain analysis of undamped systems. Journal of Engineering Mechanics- ASCE, 118, pp. 721-734, 1992. 